\title{
Silicon Carbide Sensor Systems for Harsh Environment Market Applications
}

\author{
M. Andersson ${ }^{1,2}$, B. Hammarlund ${ }^{1}$, A. Lloyd Spetz ${ }^{2}$, D. Puglisi ${ }^{2}$ \\ ${ }^{1}$ SenSiC $A B$, Isafjordsgatan $39 B$, SE-16440 Kista, Sweden \\ 2 Div. Applied Sensor Science, Dept. Physics, Chemistry and Biology, Linköping University, SE-58183 \\ Linköping, Sweden \\ mike.andersson@sensic.se
}

\begin{abstract}
The silicon carbide ( $\mathrm{SiC}$ ) processing technology for 4 inch wafers, with 6 inch in the planning phase, has reached matureness of mass production. Field effect devices based on silicon carbide, SiC-FET, employing a catalytic metal such as porous iridium (Ir) as the gate material change the current-voltage characteristics due to gas interactions with the gate metallization. Several batches of sensor devices have improved the device performance and gas response characteristics and, together with important developments on packaging of the sensors, electronics and software, sensor systems are nowadays commercially available. Here we report on Ir-gated SiC-FETs for indoor and outdoor air quality monitoring and control applications. In particular, we focus on the highest sensitivity of such sensors to ultra-low concentrations of three hazardous volatile organic compounds, i.e., benzene, naphthalene, and formaldehyde, which are commonly found in indoor environments and are considered carcinogenic already at the low parts per billion concentration range.
\end{abstract}

Key words: Silicon carbide, field effect transistor, gas sensor, air quality monitoring, ppb detection, indoor/outdoor environment

\section{Introduction}

Silicon carbide based field effect transistor (SiC-FET) technology has been developed over the last 20 years, showing very good possibilities of realizing gas sensors for high temperature and harsh environment applications such as combustion monitoring and control of car exhaust after-treatment systems [1,2]. Today, such technology has become viable for the commercial production of high volume, and price competitive products. $\mathrm{SiC}$ based gas sensors offer full functionality in a wide temperature range, harsh environments, and good resistance to thermal shock. A single sensor unit can detect several gas molecules, such as carbon monoxide (CO), ammonia $\left(\mathrm{NH}_{3}\right)$, hydrogen $\left(\mathrm{H}_{2}\right)$, and hydrocarbons $(\mathrm{HC})$ like certain volatile organic compounds (VOCs), depending on the working temperature and on the sensing material used for the gate contact. $\mathrm{SiC}$ processing technology for 4 inch wafers, with 6 inch in the planning phase, has reached matureness of mass production. The silicon carbide offer a very robust substrate platform, which even at an operating temperature over $500{ }^{\circ} \mathrm{C}$ can withstand sprinkling by water droplets, which occur e.g. in a diesel engine compartment. An ammonia sensor has been developed for control of the injection of urea in stationary engines with selective catalytic reduction (SCR) to reduce nitrogen oxide gases from diesel exhausts [3]. A sensor system with the possibility to detect the ratio between $\mathrm{CO}$ and oxygen is commercialized for control of small and medium sized bioheaters [4]. A SiC based oxygen sensor is under development to control exhaust gas recirculation (EGR) in car engines. Another SiC sensor device in research is devoted to detection of nitrogen oxide gases in car exhausts.

A 4 inch $\mathrm{SiC}$ wafer with transistors for gas sensors, designed by SenSiC $A B$ and processed at Ascatron $A B$, Sweden, is shown in Fig. 1. The chips are mounted in a holder designed to withstand harsh environment and vibration. The electronics box is also displayed.

Together with such applications for emissions and outdoor air pollution monitoring, we have developed SiC-FET gas sensors for indoor air quality $[5,6]$. Recently, Ir-gate SiC-FETs have been used for highly sensitive and selective detection of low parts per billion (ppb) concentrations of three target VOCs, i.e., formaldehyde $\left(\mathrm{CH}_{2} \mathrm{O}\right)$, naphthalene $\left(\mathrm{C}_{10} \mathrm{H}_{8}\right)$, and benzene $\left(\mathrm{C}_{6} \mathrm{H}_{6}\right)$. The sensor performance 


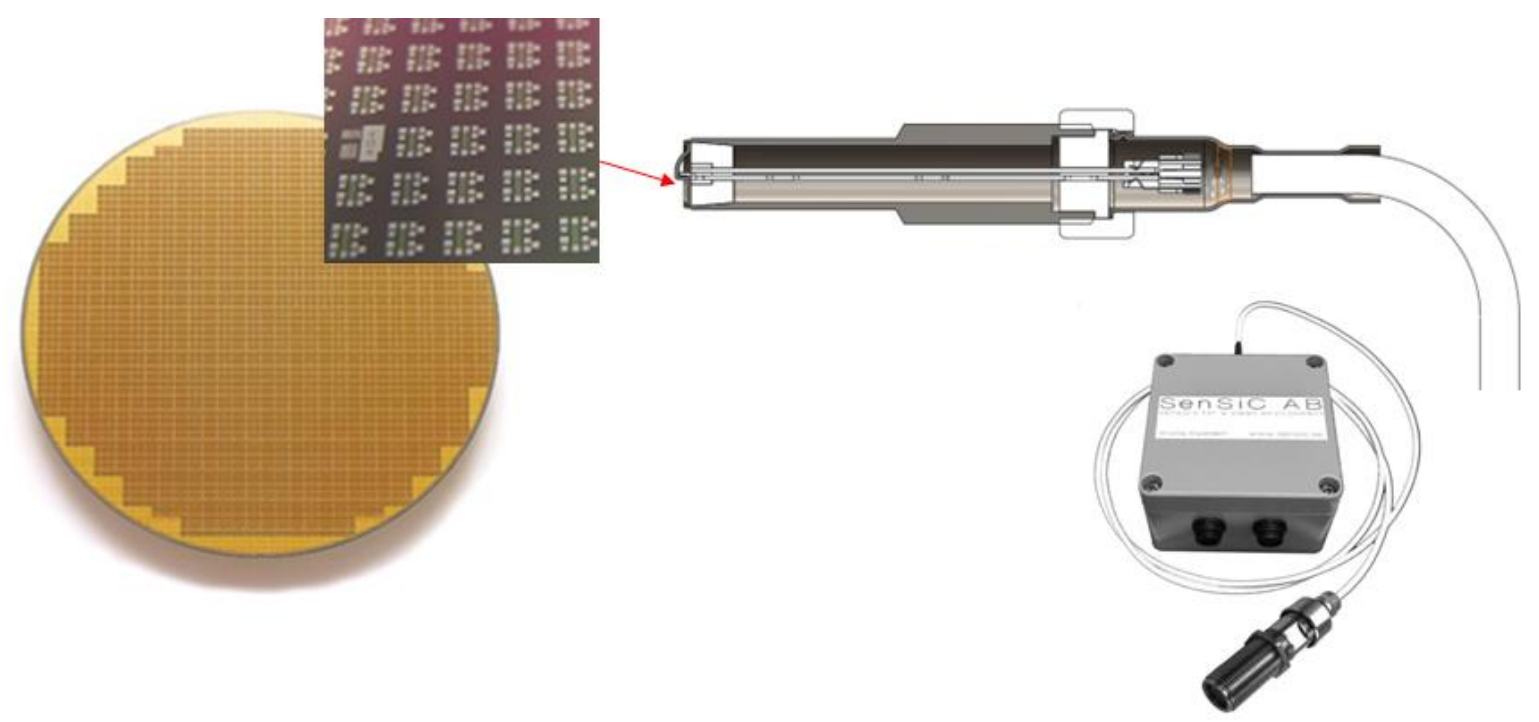

Fig. 1. A 4 inch SiC wafer with transistors for gas sensors, designed by SenSiC AB, processed at Ascatron $A B$. The chips are mounted in a holder designed to withstand harsh environment and vibration. The electronic box is also displayed.

and characteristics were investigated under controlled lab conditions at different levels of relative humidity (r.h.) up to $60 \%$, showing good stability and repeatability of the sensor response, and detection limits in the sub-ppb range [6].

The SiC-FET technology can therefore be used for both outdoor and indoor air pollution monitoring and control applications.

\section{SiC-FET gas sensor fabrication}

Two- and three-terminal depletion type metal insulator semiconductor field effect transistor (MISFET) devices were fabricated on top of 4 inch n-type $4 \mathrm{H}$-SiC wafers. Nanostructured porous Ir, used as the catalytic metal-gate contact, was deposited by DC magnetron sputtering at an Ar pressure of 50 mTorr to a total thickness of $30 \mathrm{~nm}$. The gate width is 300 $\mu \mathrm{m}$ and the corresponding gate length is $10 \mu \mathrm{m}$, with a separation between the gate and the source-drain contacts of $5 \mu \mathrm{m}$ [1]. The sensor chip, $2 \mathrm{~mm} \times 2 \mathrm{~mm}$ sized and containing four SiC-FET devices, was attached to a ceramic heater substrate (Heraeus PT 6.8/1020) together with a Pt100 temperature sensor, and mounted on a 16-pin TO8 header (see Fig. 2). Electrical connections were made using gold wire bonding.

\section{Electrical characterization}

Current-voltage (I-V) measurements were carried out at $27,100,200$, and $300{ }^{\circ} \mathrm{C}$ on several three-terminal depletion type SiC-FETs with iridium gate. A source meter Keithley 2601 was used to operate the devices sweeping the voltage $V_{D S}$ over the drain-to-source contacts from 0 to $5 \mathrm{~V}$ at a rate of $0.1 \mathrm{~V} / \mathrm{s}$, and measuring the drain current, ID. A separate gate voltage, $V_{G S}$, was applied using a stabilized voltage source. I-V characteristics were measured at zero and $2 \mathrm{~V}$ of applied $\mathrm{V}_{\mathrm{GS}}$ (see Fig. 3). As can be seen from Fig. 3, the saturation current decreases with increasing temperature, and increases with increasing gate voltage. The saturation region starts between $0.5 \mathrm{~V}$ and $1.5 \mathrm{~V}$ depending on the applied gate bias and operating temperature. It has been demonstrated that, despite that the value of the saturation current differs from

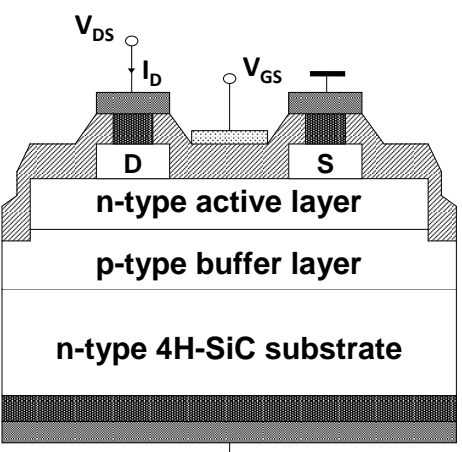

(a)

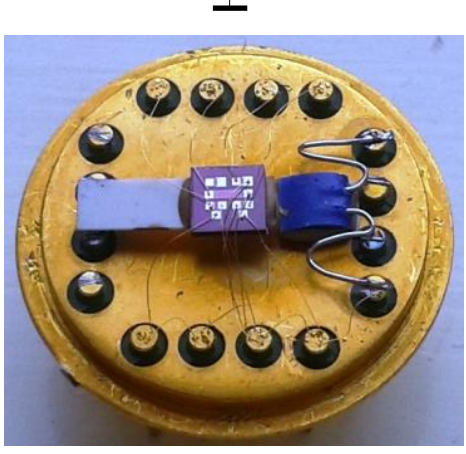

Fig. 2. (a) Cross sectional view of a threeterminal SiC-FET and (b) top view of a SiC-FET sensor chip mounted on a 16-pin TO8 header. 


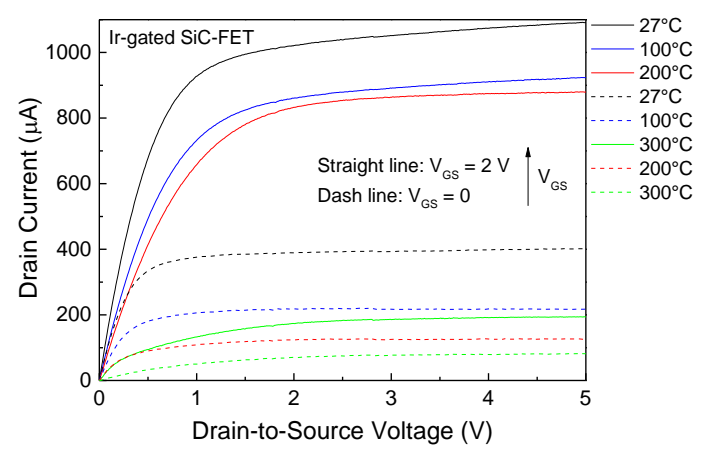

Fig. 3. Current-voltage characteristics of a threeterminal depletion type Ir-gate SiC-FET from room temperature to $300^{\circ} \mathrm{C}$ at zero and $2 \mathrm{~V}$ applied gate voltage.

sample to sample, it does not significantly affect the sensing behavior $[1,7]$.

\section{VOC tests}

Ir-gated SiC-FETs were operated at three different constant temperatures, i.e., 260, 300, and $330{ }^{\circ} \mathrm{C}$, in dry air and at different levels of relative humidity (r.h.) from 10 to $60 \%$. Benzene, naphthalene, and formaldehyde were used as target gases at several concentrations down to the sub-ppb level in order to study the sensor's sensitivity, stability, repeatability, and speed of response. The sensor devices were operated at the onset of the saturation region, keeping the drain current constant, ID, and the gate bias, $V_{G S}$, and measuring the drain-tosource voltage, $V_{D S}$, as the sensor output.

\section{Results and discussion}

A complete data analysis of the three studied VOCs at different temperatures, levels of relative humidity, and gas concentrations can be found elsewhere $[6,8]$. Figure 4 shows the sensor response to benzene at $330{ }^{\circ} \mathrm{C}$ and 60 $\%$ relative humidity (r.h.). The sensor signal

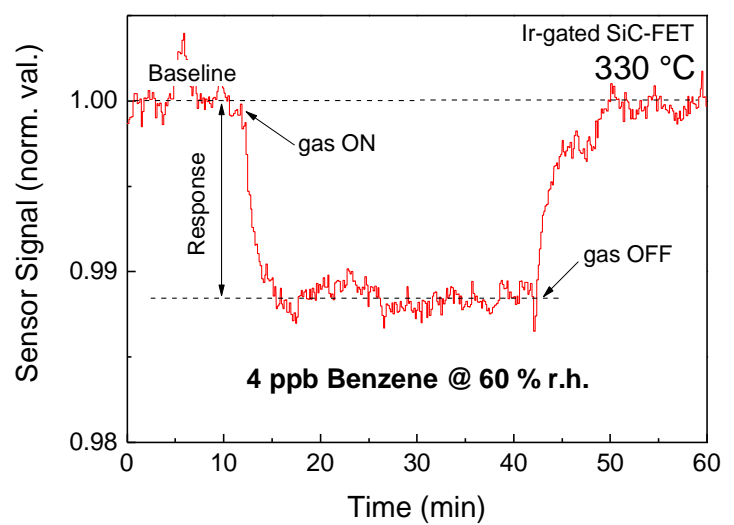

Fig. 4. Sensor response to 4 ppb benzene $\left(\mathrm{C}_{6} \mathrm{H}_{6}\right)$ at $330{ }^{\circ} \mathrm{C}$ and $60 \%$ relative humidity (r.h.). exhibits a very low electronic noise. The relative response is $1.25 \%$ at $4 \mathrm{ppb}$ (sensitivity 1.75 $\mathrm{mV} / \mathrm{ppb}$ ) with a relative error of $0.14 \%$. The response time is about $3 \mathrm{~min}$, whereas the recovery time is about $6 \mathrm{~min}$. Measurements were repeated several times at different environmental conditions for a total duration of two weeks. The presence of high humidity levels does not significantly affect the sensor performance with respect to the parameters studied. As previously reported [6], the detection limits at $60 \%$ r.h. for benzene $\left(\mathrm{C}_{6} \mathrm{H}_{6}\right)$, naphthalene $\left(\mathrm{C}_{10} \mathrm{H}_{8}\right)$, and formaldehyde $\left(\mathrm{CH}_{2} \mathrm{O}\right)$ are in the range of about $1 \mathrm{ppb}, 0.5 \mathrm{ppb}$, and 10 $\mathrm{ppb}$, respectively. These results are very promising for indoor air quality applications. The detection limits of $\mathrm{CH}_{2} \mathrm{O}$ and $\mathrm{C}_{10} \mathrm{H}_{8}$ are below the threshold limits recommended by the World Health Organization (WHO), being 81 ppb (30 min exposure) for $\mathrm{CH}_{2} \mathrm{O}$ and $1.9 \mathrm{ppb}$ (annual average) for $\mathrm{C}_{10} \mathrm{H}_{8}$ [9]. The legislation around $\mathrm{C}_{6} \mathrm{H}_{6}$ is even stricter, since no safe level of exposure are recognized for benzene [9]. Detecting levels of this VOC down to about 1 $\mathrm{ppb}$ in presence of high humidity levels is for sure an encouraging result.

Further investigation includes the development of new sensing materials to be used as the gate contact. Recently, we have combined the deposition of porous Ir with thin films of tungsten trioxide $\left(\mathrm{WO}_{3}\right)$ using pulsed laser deposition (PLD) process in collaboration with University of Oulu, Finland. From the combination of a catalytic metal and metal oxide we expect to enhance the sensor performance especially in terms of selectivity and stability during long term operation. Preliminary results are currently under evaluation.

\section{Conclusions}

SiC-FET sensor technology has demonstrated over the last 20 years to be suitable for both outdoor and indoor air quality monitoring and control applications. In this work, we have mainly focused on the most recent results obtained with Ir-gated SiC-FETs for detection of three hazardous VOCs, i.e., benzene, naphthalene, and formaldehyde, in the very low $\mathrm{ppb}$ concentration range. Iridium has shown to be a suitable material due to its high and repeatable sensitivity to the target gases upon long-term exposure at elevated temperatures and repeated gas adsorption/desorption.

\section{Acknowledgements}

The authors would like to thank the partnership of the SENSIndoor project and of the COST Action TD1105 EuNetAir. SENSIndoor has 
received funding from the European Union's Seventh Programme for research, technological development and demonstration, under grant agreement no. 604311 . The presented results were partially obtained during a Short Term Scientific Mission (STSM) hosted by Saarland University, Germany, and granted by EuNetAir. The authors would also like to thank J. Eriksson for his valuable scientific inputs and P. Möller for his technical support.

\section{References}

[1] M. Andersson, R. Pearce, A. Lloyd Spetz, New generation $\mathrm{SiC}$ based field effect transistor gas sensors, Sensors and Actuators B: Chemical 179, 95-106 (2013); doi: 10.1016/j.snb.2012.12.059

[2] M. Andersson, A. Lloyd Spetz, R. Pearce, Recent trends in silicon carbide ( $\mathrm{SiC}$ ) and graphenebased gas sensors, in: R. Jaaniso and O.K. Tan (Eds.), Semiconductor gas sensors, Woodhead Publishing Limited, 117-158 (2013); doi: 10.1533/9780857098665.2.117

[3] A. Lloyd Spetz, J. Huotari, C. Bur, R. Bjorklund, J. Lappalainen, $H$. Jantunen, A. Schuetze, M. Andersson, Chemical sensor systems for emission control from combustions, Sensors and Actuators B: Chemical 187, 184-190 (2013); doi: 10.1016/j.snb.2012.10.078
[4] www.sensic.se

[5] C. Bur, M. Bastuck, D. Puglisi, A. Schuetze, A. Lloyd Spetz, M. Andersson, Discrimination and Quantification of Volatile Organic Compounds in the ppb-Range with Gas Sensitive SiC-FETs Using Multivariate Statistics, Sensors and Actuators B: Chemical 214, 225-233 (2015); doi: 10.1016/j.snb.2015.03.016

[6] D. Puglisi, J. Eriksson, C. Bur, A. Schuetze, A. Lloyd Spetz, M. Andersson, Catalytic metal-gate field effect transistors based on SiC for indoor air quality control, Journal of Sensors and Sensor Systems 4, 1-8 (2015); doi: 10.5194/jsss-4-12015

[7] C. Bur, Selectivity Enhancement of Gas Sensitive Field Effect Transistors by Dynamic Operation, Dissertation No. 1644, Linköping University, Sweden (2015); ISBN: 978-91-7519-119-5

[8] D. Puglisi, J. Eriksson, C. Bur, A. Schuetze, A. Lloyd Spetz, M. Andersson, Silicon carbide field effect transistors for detection of ultra-low concentrations of hazardous volatile organic compounds, Materials Science Forum 778-780, 1067-1070 (2014); doi: 10.4028/www.scientific.net/MSF.778-780.1067

[9] WHO (World Health Organization): Regional Office for Europe, WHO Guidelines for Indoor Air Quality: Selected Pollutants (2010); ISBN: 978 9289002134 